\title{
Nanoscale
}

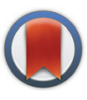

CrossMark \& click for updates

Cite this: Nanoscale, 2015, 7, 17122

\section{Excitation of Ni nanorod colloids in oscillating magnetic fields: a new approach for nanosensing investigated by TISANE}

\author{
Philipp Bender, ${ }^{*} \dagger^{\mathrm{a}}$ Annegret Günther, ${ }^{\mathrm{b}}$ Dirk Honecker, $+^{\mathrm{b}}$ Albrecht Wiedenmann, ${ }^{\mathrm{c}}$ \\ Sabrina Disch, ${ }^{\star d}$ Andreas Tschöpe, ${ }^{a}$ Andreas Michels ${ }^{b}$ and Rainer Birringer ${ }^{a}$
}

\begin{abstract}
The response of a colloidal dispersion of Ni nanorods to an oscillating magnetic field was characterized by optical transmission measurements as well as small-angle neutron scattering (SANS) experiments using the TISANE (Time-dependent SANS experiments) technique. Exposed to a static magnetic field, the scattering intensity of the rod ensemble could be well described by the cylinder form factor using the geometrical particle parameters (length, diameter, orientation distribution) determined by transmission electronmicroscopy and magnetometry. An oscillation of the field vector resulted in a reorientation of the nanorods and a time-dependency of the scattering intensity due to the shape anisotropy of the rods. Analysis of the SANS data revealed that in the range of low frequencies the orientation distribution of the rods is comparable to the static case. With increasing frequency, the rod oscillation was gradually damped due to an increase of the viscous drag. It could be shown that despite of the increased friction in the high frequency range no observable change of the orientation distribution of the ensemble with respect to its symmetry axis occurs.
\end{abstract}

Received 25th June 2015, Accepted 9th September 2015 DOI: $10.1039 / \mathrm{c} 5 \mathrm{nr} 04243 \mathrm{~g}$ www.rsc.org/nanoscale ments. ${ }^{14}$ The phase lag between the particle magnetic moments with respect to the rotating field vector is determined as a function of rotation frequency, either by measuring the macroscopic magnetic moment of the ensemble ${ }^{14}$ or, in case of anisometric particles like nanorods, its optical extinction. ${ }^{5,19,20}$ In both cases the measured parameter - magnetic moment $m \propto\langle\cos \Theta\rangle$, transmitted light intensity $I \propto\left\langle\cos ^{2} \Theta\right\rangle-$ is an ensemble average which depends on the distribution $P(\Theta)$ of the angle $\Theta$ between the moments and the field vector. However, continuous rotation of magnetic nanoparticles is restricted to purely viscous matrices. To enable measurements of general viscoelastic matter, the application of oscillating magnetic fields has been proposed. ${ }^{6}$ In ref. 6 single domain uniaxial ferromagnetic $\mathrm{Ni}$ nanorods $\mathrm{s}^{22-25}$ were used as probe particles and stimulated to an oscillating motion by an oscillation of the magnetic field vector with constant magnitude. Analysis of the frequency-dependent response of the colloidal dispersion was employed to determine the viscosities as well as the shear moduli of surrounding viscoelastic matrices on the nanoscale. For data analysis it was assumed that the orientation distribution $P(\Theta)$ of the nanorods corresponds to the static state even in the high-frequency range. However, in the particular case of rotating magnetic fields with constant magnitude, numerical simulations come to the conclusion that the orientation distribution $P(\Theta)$ of ferromagnetic nanorods not only depends on the magnitude of the field vector but also on 
the frequency $\omega .{ }^{26}$ It can be shown that $P(\Theta)$ evolves from the static state at a given field strength with increasing frequency $\omega \rightarrow \infty$ towards an isotropic orientation distribution. ${ }^{26}$ Moreover numerical simulations come to the conclusion that the orientation distribution of magnetic particles exposed to static magnetic fields can be significantly altered by shear-flows and becomes in most cases asymmetric. ${ }^{27-30}$ Hence, the question arises whether the orientation distribution $P(\Theta)$ of magnetic dipoles in oscillating magnetic fields is also frequencydependent.

In the current study an ensemble of Ni nanorods dispersed in water was exposed to oscillating magnetic fields with constant magnitude $\left(\mu_{0} H=6 \mathrm{mT}\right)$ and varying frequency $(\omega=$ 0-18849.6 Hz). The evolution of the orientation distribution of the colloid was monitored by analyzing time-resolved SANS patterns. In order to achieve a sufficiently high time-resolution the novel TISANE-mode (Time-dependent SANS experiments) was employed, which was recently installed at the instrument D22 at the Institut Laue Langevin (ILL), Grenoble. The goal was to test if the orientation distribution remains comparable to the static state. This is an essential condition so that the relaxation behavior detected by means of ensemble averaging methods (e.g. magnetization and optical transmission measurements) can be analyzed by mean field approaches. ${ }^{6,26}$

\section{Sample}

\section{A. Synthesis}

The synthesis of colloidal dispersions of $\mathrm{Ni}$ nanorods involves several steps as described in detail in ref. 5. Briefly, the nanorods for this sample were prepared by current-pulsed electrodeposition of $\mathrm{Ni}^{31}$ from a Watts-bath ${ }^{32}$ into a nanoporous alumina template, which was prepared by a twofold anodization process. ${ }^{33}$ The rods were released from the template by dissolving the alumina in a $20 \mathrm{mM}$ sodium hydroxide solution, which additionally contained polyvinyl-pyrrolidone (PVP) as surfactant. The PVP adsorbed on the rod surface and acted as steric stabilizer to prevent agglomeration during the following washing process which consisted of multiple centrifugation and redispersion steps in deuterium oxide $\left(\mathrm{D}_{2} \mathrm{O}\right)$. The final dispersion of rods in $\mathrm{D}_{2} \mathrm{O}$ had a total volume of $\approx 500 \mu \mathrm{l}$.

\section{B. Structural characterization}

The structure of the rods was characterized by Transmission Electron Microscopy (TEM, JEOL JEM-2011, Fig. 1). The nanorods had a cylindrical shape and analysis of $N=100$ particles revealed an average length $l=170 \pm 4 \mathrm{~nm}\left(\sigma_{1}=40 \mathrm{~nm}\right)$ and average diameter $d=23.8 \pm 0.2 \mathrm{~nm}\left(\sigma_{\mathrm{d}}=2 \mathrm{~nm}\right)$. Using the volume of equally sized cylinders and the saturation magnetization of bulk Ni $\left(M_{\mathrm{S}}\right.$ $=4.85 \times 10^{5} \mathrm{~A} \mathrm{~m}^{-1}$ (ref. 34)), the average magnetic moment of the rods can be estimated to $m_{\mathrm{r}}=3.7 \times 10^{-17} \mathrm{Am}^{2}$.

\section{Magnetic characterization}

To characterize the magnetization behavior of the rod ensemble dispersed in $\mathrm{D}_{2} \mathrm{O}$, the quasistatic magnetization curve of

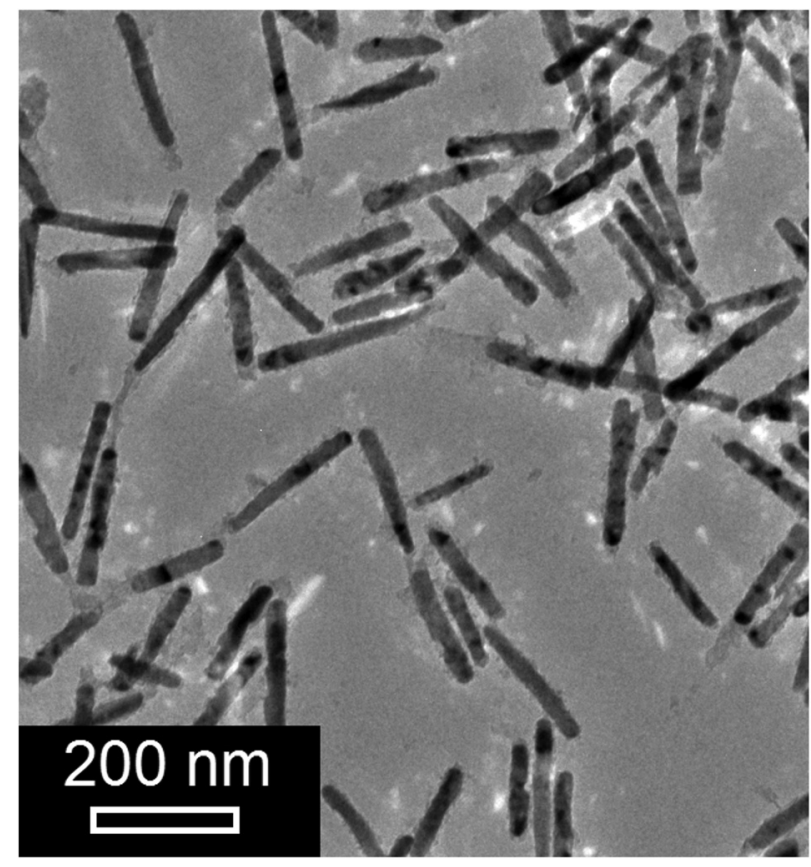

Fig. 1 TEM micrograph of the Ni nanorods.

$V=135 \mu \mathrm{l}$ of the colloidal dispersion was measured at $T=$ $295 \mathrm{~K}$ with a Vibrating Sample Magnetometer (VSM, Lakeshore 7300). With diameters below about $40 \mathrm{~nm}$ the nanorods are expected to be single-domain particles, which are magnetized along their long-rod axes due to shape anisotropy. ${ }^{2-25}$ Dispersed in a liquid matrix the nanorods align preferentially parallel to a static external magnetic field, ${ }^{5,35}$ giving rise to the pseudo-superparamagnetic behavior observed in Fig. 2. The curve could be reasonably well described by a single Langevin

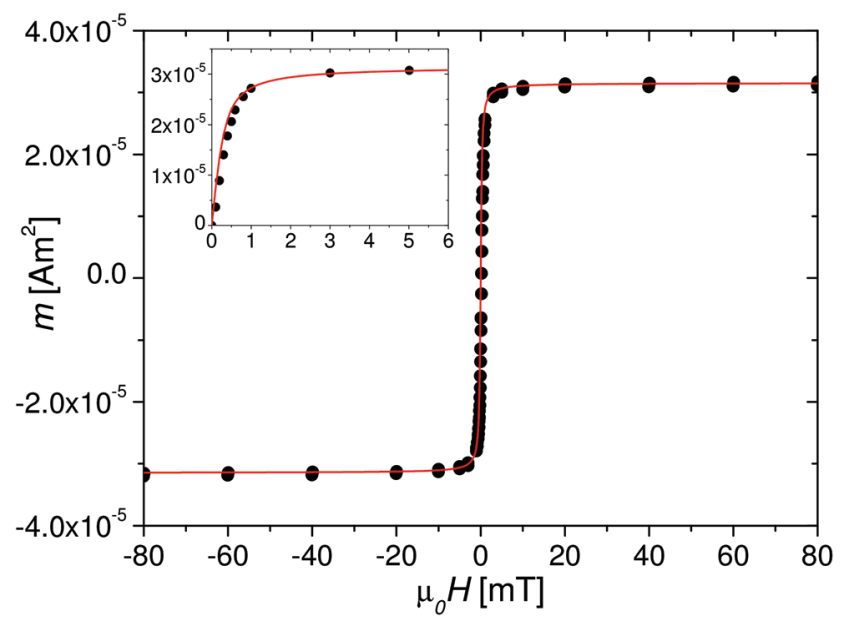

Fig. 2 Magnetization curve of the nanorod dispersion in $\mathrm{D}_{2} \mathrm{O}$ and corresponding fit with a single Langevin function (red line) with the magnetic moment $m_{\mathrm{r}}=3 \times 10^{-17} \mathrm{Am}^{2}$ of the nanorods and the saturation moment $m_{\mathrm{S}}=3.2 \times 10^{-5} \mathrm{Am}^{2}$ of the colloidal dispersion; inset shows the upper right quadrant from $0 \mathrm{mT}$ to $6 \mathrm{mT}$. 
function $m(H)=m_{\mathrm{S}} L(\xi)=m_{\mathrm{S}}(\operatorname{coth}(\xi)-1 / \xi)$ with $\xi=m_{\mathrm{r}} \mu_{0} H / k_{\mathrm{B}} T$, where $\mu_{0}=4 \pi \times 10^{-7} \mathrm{~V} \mathrm{~s}(\mathrm{Am})^{-1}$ is the vacuum permeability and $k_{\mathrm{B}}=1.381 \times 10^{-23} \mathrm{~J} \mathrm{~K}^{-1}$ the Boltzmann constant. The best fit result was obtained for a saturation moment $m_{\mathrm{S}}=3.2 \times 10^{-5}$ $\mathrm{Am}^{2}$ and an average magnetic moment of $m_{\mathrm{r}}=3 \times 10^{-17} \mathrm{Am}^{2}$. The moment is below the average rod moment estimated from TEM, which can be attributed to a $\approx 1.5 \mathrm{~nm}$ thick oxide layer. $^{36,37}$ From the saturation moment of the colloid the volume concentration of magnetic phase can be estimated to $V_{\text {mag }} / V=0.5 \%$. Additionally, the orientation of the rods at a given field strength can be extracted from the quasistatic magnetization behavior. ${ }^{38}$ The orientation distribution $P(\Theta)$ is symmetric around the field axis and can be represented by a tapered distribution with fixed characteristic polar angle between the moments and the field vector $[\Theta(H)]=\arccos (L(H))$. According to the magnetization measurement (Fig. 2) the Langevin function takes on a value of $L=0.978$ at $\mu_{0} H=6 \mathrm{mT}(\xi=44)$, and hence the angle between the rod axes and the magnetic field vector is $[\Theta]=12^{\circ}$. This value is important for the analysis of the optical transmission (OT) and SANS experiments where the response of the colloid to an oscillating field with magnitude $\mu_{0} H=6 \mathrm{mT}$ was characterized.

\section{Nanorod colloids in oscillating fields}

\section{A. Experimental setups}

1. Optical transmission OT. The experimental setup used for the OT-measurements is described in detail in ref. 6 . Briefly, an optical grade glass cuvette containing the colloidal dispersion was placed in the center of two crossed pairs of Helmholtz-coils. The nanorod oscillation was monitored by detecting the time-dependent transmitted light intensity of linearly polarized light, passed through the cuvette. To achieve an optimal resolution of the oscillating light intensity the original dispersion was diluted by a factor of 100 , reducing the volume concentration of the nanorods in the dispersion from $V_{\text {mag }} / V=$ $0.5 \%$ o to $V_{\mathrm{mag}}^{\mathrm{OT}} / V=0.005 \%$.
2. Small angle neutron scattering. For the SANS experiments $\approx 300 \mu \mathrm{l}$ of the original colloidal dispersion of nanorods was filled into a standard quartz cuvette (Hellma) and mounted with a silicon holder in the center of two crossed Helmholtz coil-sets (Fig. 3). Static fields could be generated by connecting the coils to a DC power supply (Agilent 6642A). In case of AC-fields, the coils were driven by a resonance circuit consisting of a frequency generator (Agilent 33220A) and an audio amplifier (Crown XLS5000). To match the impedance of the coils at high frequencies, a variable number of capacities connected to a 100 pin terminal block (Agilent U2903A) could be connected in series by means of a Digital I/O (Agilent U2653A).

The time-resolved SANS experiments were performed on the instrument D22 at the ILL, Grenoble, using the newly implemented TISANE technique. The time-resolution in SANS experiments using a continuous neutron beam is generally limited to some ms due to frame overlap related to the wavelength broadening. The TISANE technique ${ }^{39}$ is a stroboscopic method, which allows to study fast cyclic processes down to the microsecond regime, where the neutron beam is chopped in neutron bunches of frequency $\omega_{\mathrm{n}}$ by means of a chopper located close to the monochromator in front of the collimation section. For this experiment unpolarized neutrons with a mean wavelength of $\lambda=6 \AA$ and a wavelength spread of $\Delta \lambda / \lambda=$ $10 \%$ were used. The distance between chopper (C) and sample (S) was $L_{1}=20.88 \mathrm{~m}$ (Fig. 3) and the area detector (D) with $128 \times 128$ pixels of $8 \times 8 \mathrm{~mm}^{2}$ was placed $L_{2}=8 \mathrm{~m}$ behind the sample. The data acquisition was triggered with a frequency generator at given frequency $\omega_{\mathrm{d}}$ and one period was divided in 100 time channels with a channel width of $t=2 \pi /\left(100 \times \omega_{\mathrm{d}}\right)$. For optimal time resolution, the frequency of the oscillating field (sample frequency $\omega_{\mathrm{s}}$ ), the chopper frequency $\left(\omega_{\mathrm{n}}\right)$ and the data acquisition frequency $\left(\omega_{\mathrm{d}}\right)$ must satisfy the conditions ${ }^{39,40}$

$$
\omega_{\mathrm{d}}=\omega_{\mathrm{s}}-\omega_{\mathrm{n}} \text { with }: \omega_{\mathrm{n}}=\omega_{\mathrm{s}} \frac{L_{2}}{L_{1}+L_{2}} .
$$

The rawdata treatment was carried out by means of the GRASP software package. ${ }^{41}$

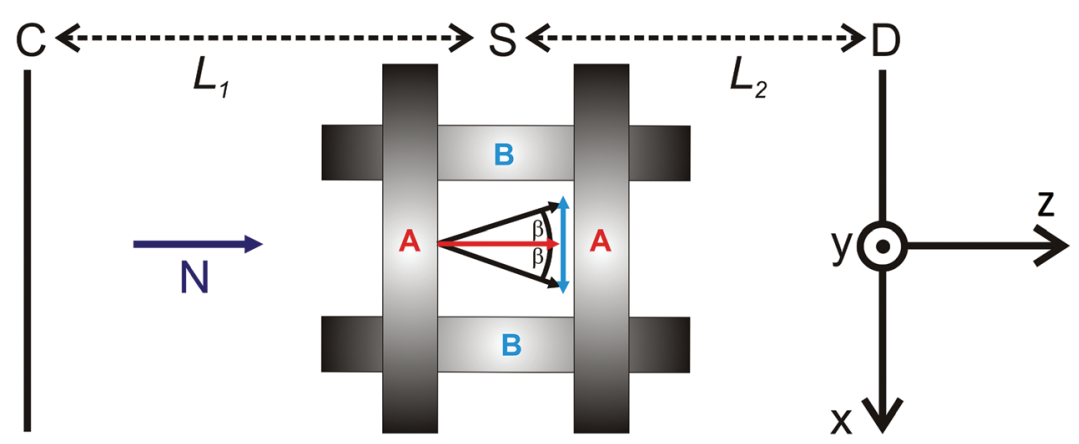

Fig. 3 Schematical illustration of the topview of the coil-setup used for the time-resolved SANS (TISANE) experiments. With coil pair A a static field of $6 \mathrm{mT}$ was applied parallel to the pulsed neutron beam N ( $z$-direction). With coil pair $B$ an alternating field with amplitude $1.2 \mathrm{mT}$ was generated perpendicular ( $x$-direction) to the static field, resulting in an oscillation of the field vector $\beta(t)$ in the $x-z$-plane. 


\section{B. Measurement protocol}

For both, the OT- as well as SANS-measurements, the response of the colloid to oscillating magnetic fields with magnitude $\mu_{0} H \approx 6 \mathrm{mT}$ was monitored as a function of the frequency $\omega_{\mathrm{s}}$. The fields were generated by applying a static field of $6 \mathrm{mT}$ (with coil pair A in $z$-direction, Fig. 3) and an alternating field with magnitude $1.2 \mathrm{mT}$ and frequency $\omega_{\mathrm{s}}$ perpendicular to it (with coil pair B in $x$-direction, Fig. 3 ). Due to the small amplitude of the excitation field the magnitude of the field vector can be considered as constant $\left(\mu_{0} H=6 \pm 0.12 \mathrm{mT} \approx 6 \mathrm{mT}\right)$. The angular position of the magnetic field vector in the $x-z$ plane is hence $\beta(t)=\beta_{0} \exp \left(i \omega_{\mathrm{s}} t\right)$, with $\beta_{0}=\arctan (1.2 / 6)=$ $11.3^{\circ}$. The field stimulated an oscillation $\zeta(t)=\zeta_{0}\left(\omega_{\mathrm{s}}\right) \exp \left(i\left(\omega_{\mathrm{s}} t\right.\right.$ $\left.\left.+\phi\left(\omega_{\mathrm{s}}\right)\right)\right)$ of the nanorods, with the frequency-dependent amplitude $\zeta_{0}\left(\omega_{\mathrm{s}}\right)$ and phase shift $\phi\left(\omega_{\mathrm{s}}\right)$ between the nanorod axes and the field vector. In case of the OT-measurements the phase shift $\phi\left(\omega_{\mathrm{s}}\right)$ as well as the oscillation amplitude $\zeta_{0}\left(\omega_{\mathrm{s}}\right)$ were extracted from the oscillating transmitted light intensity as described in ref. 6, under the assumption that all nanorods are mutually aligned. The results of both quantities obtained from optical transmission are presented in the following section and used as the basis for the analysis of the SANSexperiments.

\section{Experimental results}

1. Optical transmission (OT). In Fig. 4 the phase shift between the nanorods and the field vector as well as the magnitude of the oscillation amplitude of the nanorods is shown. For frequencies $\omega_{\mathrm{s}}<1800 \mathrm{~Hz}$ the phase shift only slightly increased from $\phi=0^{\circ}$ to $6^{\circ}$ and the oscillation amplitude $\zeta_{0}$ equaled $\beta_{0}=11.3^{\circ}$ in that range. Hence, in this frequency region the nanorod oscillation can be regarded as to be in

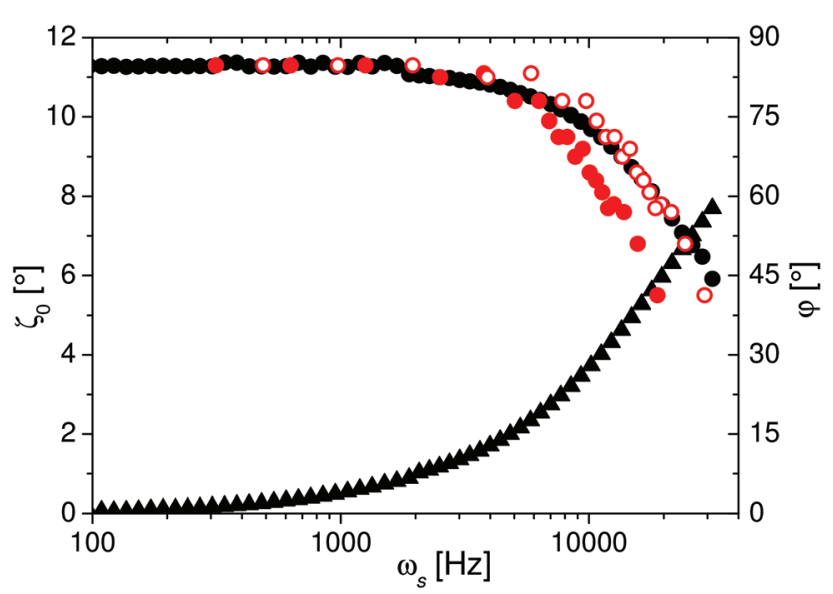

Fig. 4 Magnitude of the oscillation amplitude of the nanorods $\zeta_{0}$ (black circles) and the phase shift $\phi$ (black triangles) between their axes and the oscillating field vector as a function of frequency, determined by OT. The red circles are the magnitudes of the oscillation amplitudes determined by SANS. Good agreement with the results from OT is obtained, when the values are shifted by a factor of 1.5 on the frequency-axis (open red circles). phase with the field vector. With increasing frequency however, the phase shift increased gradually up to $\phi=57.7^{\circ}$ at $\omega_{\mathrm{s}}=31415.9 \mathrm{~Hz}$, due to the growing viscous friction between the nanorods and the surrounding water. As a direct result, the oscillation amplitude of the nanorod ensemble decreased and reached a value of $\zeta_{0}=5.9^{\circ}$ at $\omega_{\mathrm{s}}=31415.9 \mathrm{~Hz}$. From the frequency-dependent response of the nanorod ensemble the viscoleastic properties of the surrounding matrix can be extracted. ${ }^{6}$ For that purpose the imaginary part of the complex response function $\hat{X}\left(\omega_{\mathrm{s}}\right)$ is determined from the experimental data (Fig. 4) as $X^{\prime \prime}\left(\omega_{\mathrm{s}}\right)=\zeta_{0}\left(\omega_{\mathrm{s}}\right) / \beta_{0} \sin \phi$ (Fig. 5). Theoretically it is found that ${ }^{6}$

$$
X^{\prime \prime}\left(\omega_{\mathrm{s}}\right)=\frac{\omega_{\mathrm{s}} / \omega_{\mathrm{c}}}{1+\left(\omega_{\mathrm{s}} / \omega_{\mathrm{c}}\right)^{2}}
$$

in a purely viscous matrix, with the characteristic relaxation frequency $\omega_{\mathrm{c}}$. In an oscillating field with the Langevin-parameter $\xi$ the characteristic relaxation frequency $\omega_{\mathrm{c}}=\omega_{\mathrm{B}} \xi / 2$ is related to the Brownian relaxation time $\omega_{\mathrm{B}} \cdot{ }^{42}$ Hence, with the parameters used in this study $(\xi=44)$ the characteristic frequency $\omega_{\mathrm{c}}$ is larger by a factor 22 compared to the Brownian relaxation frequency $\omega_{\mathrm{B}}$. The mean value of the characteristic relaxation frequency of the nanorods can be obtained by a fit of the experimentally determined imaginary part $X^{\prime \prime}\left(\omega_{\mathrm{s}}\right)$ of the response function with eqn (2), using a logarithmic normal distribution of $\omega_{\mathrm{c}}$ :

$$
f\left(\omega_{\mathrm{c}}\right)=\frac{1}{\omega_{\mathrm{c}} \sigma \sqrt{2 \pi}} \exp \left(-\frac{\left(\ln \omega_{\mathrm{c}}-\ln \omega_{\mathrm{c}}^{0}\right)^{2}}{2 \sigma^{2}}\right) .
$$

The best fit result shown in Fig. 5 was obtained for $\omega_{\mathrm{c}}{ }^{0}=$ $21460 \mathrm{~Hz}$ and the scatter parameter $\sigma=0.58$, and thus the mean value of the characteristic frequency is $\left\langle\omega_{\mathrm{c}}\right\rangle=$ $\omega_{\mathrm{c}}{ }^{0} \exp \left(\sigma^{2} / 2\right)=25391 \mathrm{~Hz}$.

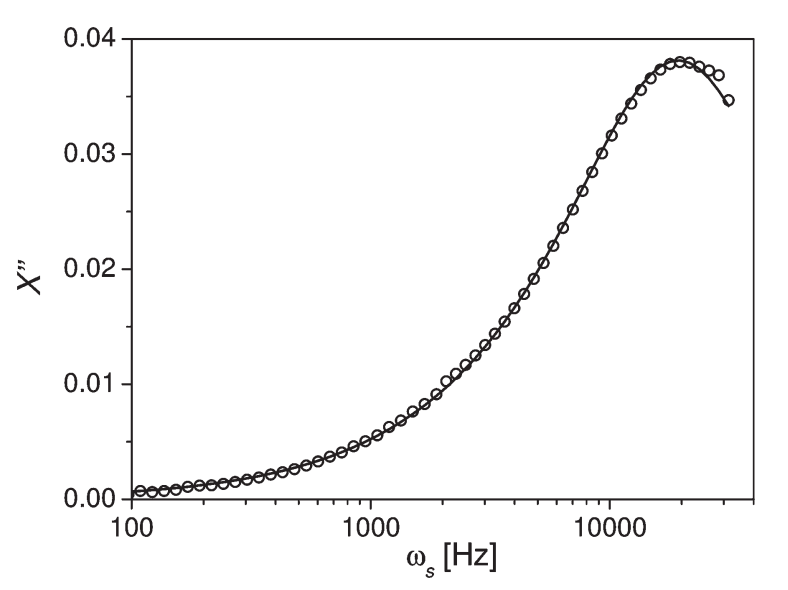

Fig. 5 Imaginary part $X$ " of the response function of the nanorods as a function of frequency, determined by OT (open black circles). The black line is the fit with eqn (2) assuming a lognormal-distribution (eqn (3)) of the characteristic relaxation frequency $\omega_{\mathrm{c}}$ with $\omega_{\mathrm{c}}{ }^{0}=21460 \mathrm{~Hz}$ and $\sigma=$ 0.58 . 
Determination of the frequency-dependent response function from the time-dependent transmitted light intensity $I(t) \propto$ $\left\langle\cos ^{2} \Theta(t)\right\rangle$ is based on the transmitted light intensity $I \propto$ $\left\langle\cos ^{2} \Theta\right\rangle$ in the static state. Implicitly it is thus assumed that the orientation distribution $P(\Theta)$ in the dynamic case corresponds to the static state, despite the distribution of relaxation frequencies and the growing viscous friction with increasing frequency. To gain information about the dynamic orientation distribution of ensembles of dipoles in time-modulated fields from OT- or magnetization measurements the experimental data has to be modeled with the Fokker-Planck-equation, as done e.g. in case of rotating fields. ${ }^{26}$ In contrast, a direct experimental access to the frequency-dependent orientation distribution of the individual particles is provided by the analysis of the time-dependent 2D SANS scattering patterns, as shown in the following.

2. SANS. As a starting point, the scattering behavior of the nanorod ensemble in a static magnetic field of $\mu_{0} H=6 \mathrm{mT}$, applied parallel to the neutron beam with coil pair A (Fig. 3), was characterized. Fig. 6 shows the 2D scattering pattern and Fig. 7 displays the SANS intensity as a function of the magnitude of the scattering vector $Q$ after circular averaging. In order to retrieve information about the orientation distribution of the nanorods from the scattering data, the experimental results were simulated.

In general the unpolarized SANS intensity $I(\vec{Q})$ of a dilute particle system is given by ${ }^{43}$

$$
I(\vec{Q})=n \Delta \rho^{2} V^{2}|F(\vec{Q})|^{2} .
$$

Here $n$ is the number density of particles, $\Delta \rho$ the scattering length density difference between particle and solvent, $V$ the volume of the particle and $F(\vec{Q})$ the form factor. Please note that in the following magnetic scattering contributions will be neglected due to the significantly smaller magnetic scattering length density contrast $\Delta \rho_{\mathrm{m}}{ }^{2}$ compared to the nuclear scatter-

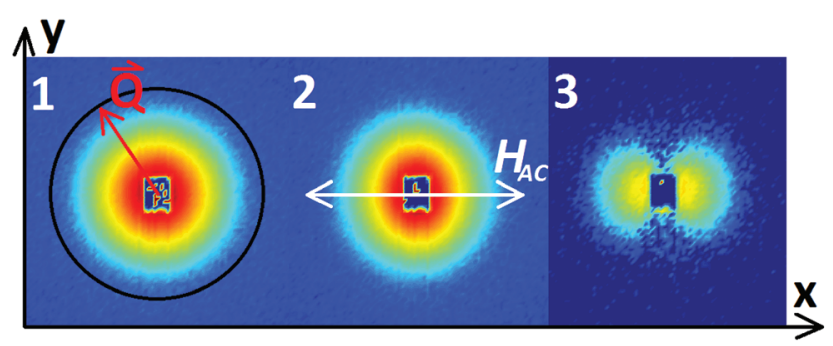

Fig. 6 (1) $2 \mathrm{D}$ scattering pattern $I(\vec{Q})$ of the colloid exposed to a static magnetic field of $6 \mathrm{mT}$ in $z$-direction. (2) Summation of the time-dependent scattering patterns of the colloid in an oscillating field with frequency $\omega_{\mathrm{s}}=157.1 \mathrm{~Hz}$. To generate the oscillating field an alternating field $H_{\mathrm{AC}}(t)=H_{\mathrm{AC}}{ }^{0} \sin (\omega t)$ with amplitude $\mu_{0} H_{\mathrm{AC}}{ }^{0}=1.2 \mathrm{mT}$ was applied in $x$-direction. (3) Scattering pattern of the colloid exposed to the oscillating field (2) substracted from scattering pattern of the colloid exposed to the static field (1). The difference along the $y$-axis is $\approx 0$ over the whole $Q$-range, but significantly above 0 in $x$-direction.

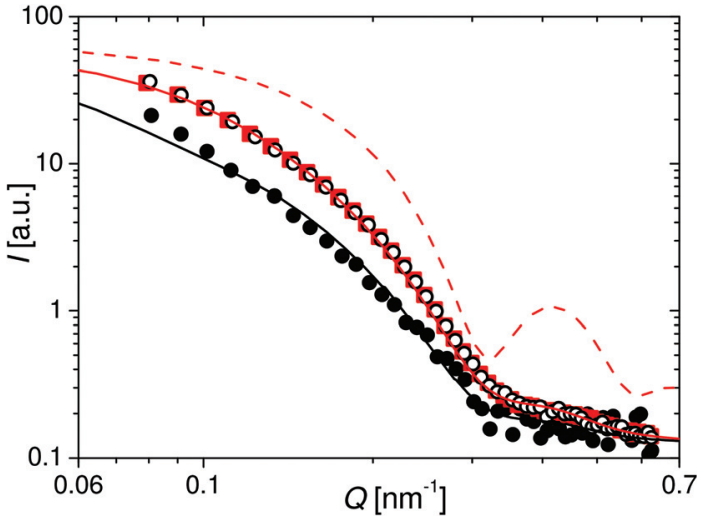

Fig. 7 Red squares: SANS intensity $I(Q)$ after circular averaging the 2D scattering pattern from Fig. 6 of the colloid in the static magnetic field. Red line: simulated intensity for an ensemble of $\mathrm{Ni}$ cylinders with the length and diameter distributions determined by TEM oriented parallel to the neutron beam (dashed) and with an angle of $\Theta=12^{\circ}$ between the cylinder axes and the neutron beam oriented symmetrically around the symmetry axis (straight). Black open circles: SANS intensity in the vertical sector of a random time channel of the time-resolved measurement for $\omega_{\mathrm{s}}=157.1 \mathrm{~Hz}$. Black full circles: SANS intensity in the horizontal sector of the time channel with minimal integrated intensity of the timeresolved measurement for $\omega_{\mathrm{s}}=157.1 \mathrm{~Hz}$. Black line: simulated intensity for an ensemble of $\mathrm{Ni}$ cylinders with the length, diameter and orientation distributions determined by TEM and VSM, rotated $\zeta=11.3^{\circ}$ out of the neutron beam.

ing length density contrast $\Delta \rho_{\mathrm{n}}{ }^{2} \approx 6.6 \Delta \rho_{\mathrm{m}}{ }^{2}$ of the nanorods dispersed in $\mathrm{D}_{2} \mathrm{O} .{ }^{44}$ The geometrical particle form factor of a cylinder with volume $V$, radius $r$ and length $l$ is given by ${ }^{43,45,46}$

$$
F(Q, \mu)=\frac{2 J_{1}\left(Q r \sqrt{1-\mu^{2}}\right)}{Q r \sqrt{1-\mu^{2}}} \frac{\sin (Q l \mu / 2)}{Q l \mu / 2},
$$

with $J_{1}(x)$ being the first-order Bessel function and $\mu$ the normalized projection of the oriented cylinder on the detector plane. With the orientation angles $\Theta$ and $\Phi$ (Fig. 8), the projection of the cylinder is $\mu=\sin \Theta$ and the contributions in $x$ - and $y$-direction are

$$
\mu_{x}=\sin \Theta \cos \Phi, \mu_{y}=\sin \Theta \sin \Phi .
$$

To simulate the scattering data, a non-interacting ensemble of $\mathrm{Ni}$ cylinders dispersed in $\mathrm{D}_{2} \mathrm{O}$ was assumed, taking into account the length and diameter distributions determined by TEM (minus the oxide layer). As shown in Fig. 7, the simulated intensity $I(Q)$ of such a cylinder ensemble aligned parallel to the field vector and hence to the neutron beam $\left(\Theta=0^{\circ}\right)$ shows significant differences compared to the experimental data, which indicates a significant orientation distribution in the experiment at Langevin-parameter $\xi=44$. According to the quasistatic magnetization measurements, at a field strength of $\mu_{0} H=6 \mathrm{mT}$ the characteristic angle between the rod moments and the field vector was 


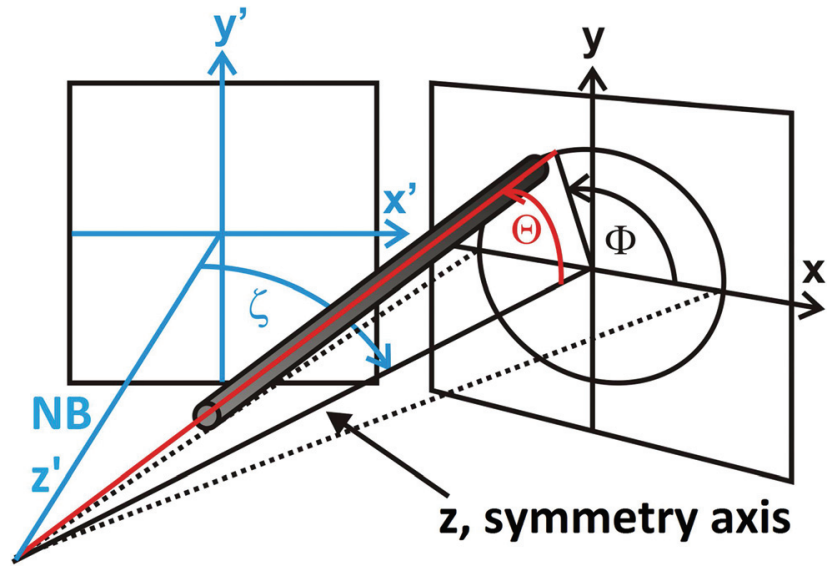

Fig. 8 Schematical illustration of the orientation of an individual nanorod with respect to the detector and definition of the relevant angles. When the ensemble is quasistatically rotated by the angle $\zeta$ out of the neutron beam (NB, $z^{\prime}$-direction) the $z$-direction is the symmetry axis of the ensemble.

$[\Theta]=12^{\circ}$. To include an orientation distribution the orientation average

$$
F(\vec{Q})=\frac{\int_{0}^{2 \pi} \mathrm{d} \Phi \int_{0}^{\pi} \mathrm{d} \Theta \sin \Theta P(\Phi, \Theta) F(Q, \mu)}{\int_{0}^{\pi} \mathrm{d} \Theta \sin \Theta P(\Phi, \Theta)} .
$$

has to be computed. For the simulation it was assumed that the distribution is symmetric in $\Phi$ around the $z$-axis so that $P(\Phi, \Theta)=P(\Theta) / 2 \pi$ (Fig. 8).

As shown in Fig. 7, the simulated intensity is in very good agreement with the experimental data when the polar angle is fixed at $\Theta=12^{\circ}\left(P(\Theta)=\delta\left(\Theta-12^{\circ}\right)\right)$. Hence, the analysis of the SANS data in the static field agrees with the results of the magnetization measurements. In the following the cylinder model will be applied in order to extract the dynamic orientation distribution of the nanorod ensemble in oscillating magnetic fields from the time-dependent scattering patterns.

Application of the alternating excitation field in $x$-direction (Fig. 6) resulted in an oscillation of the field vector in the $x-z$ plane, stimulating an oscillation of the nanorod ensemble. In case of a rotation of the nanorods exclusively in the $x-z$-plane by the angle $\zeta$, the orientation angles with respect to the neutron beam (in the following the $z^{\prime}$-direction, as defined in Fig. 8) are

$$
\begin{aligned}
& \Theta^{\prime}=\arccos (\cos \Theta \cos \zeta-\sin \Theta \cos \Phi \sin \zeta) \\
& \Phi^{\prime}=\arccos \left(\frac{\sin \Theta \cos \Phi \cos \zeta+\cos \Theta \sin \zeta}{\sin \Theta^{\prime}}\right)
\end{aligned}
$$

and hence the projections of the cylinder (eqn. (6)) on the detector $\left(x^{\prime}-y^{\prime}\right.$-plane) are

$$
\begin{gathered}
\mu_{x^{\prime}}=\sin \Theta^{\prime} \cos \Phi^{\prime}=\sin \Theta \cos \Phi \cos \zeta+\cos \Theta \sin \zeta \\
\mu_{y^{\prime}}=\sin \Theta^{\prime} \sin \Phi^{\prime}=\sin \Theta \sin \Phi .
\end{gathered}
$$

Thus, the SANS intensity $I(Q)$ along the $y^{\prime}$-direction does not change (compare eqn (6)) but tends to decrease along the $x^{\prime}$-direction with increasing rotation (Fig. 6). In order to evaluate the anisotropic scattering patterns, analysis of the timedependence of the nanorod oscillation was performed by integrating the intensity in $\pm 10^{\circ}$ sectors parallel to the alternating field as well as perpendicular to it, in each case for all 100 time channels.

Fig. 9 shows three examples of the integrated intensities detected for sample frequencies of $\omega_{\mathrm{s}}=157.1 \mathrm{~Hz}, \omega_{\mathrm{s}}=11309.7$ $\mathrm{Hz}$ and $\omega_{\mathrm{s}}=18849.6 \mathrm{~Hz}$. For each frequency the intensity in the vertical sector is constant and corresponds to the radiallyaveraged scattering intensity of the sample in the static field (normalized intensity $\sim 1$ ), whereas a significant modulation is observed in the horizontal sector. This observation verifies that the nanorod oscillation exclusively occurs in the $x-z$ plane. With increasing frequency the minimum of the timedependent integrated intensity (at $\omega_{\mathrm{s}} t=90^{\circ}$, Fig. 9) shifts to larger values and can be explained by a damping of the nanorod oscillation due to the increasing viscous drag. In order to extract the magnitude of the average oscillation amplitude of the nanorod ensemble in analogy to the OT-measurements, the SANS measurement with the lowest sample frequency $\omega_{\mathrm{s}}=157.1 \mathrm{~Hz}$ was used as reference. In this case it is safe to assume that the orientation distribution of the nanorod ensemble remains symmetric and rotates in phase with the field vector, i.e. the rotation angle $\zeta(t)$ of the symmetry axis of the nanorod ensemble equals the angle $\beta(t)$ of the field vector at a given time channel $\omega_{\mathrm{s}} t$ (Fig. 9).

To correlate the integrated intensity with the rotation angle $|\zeta(t)|$, the inverse function, i.e. $|\beta(t)|$ as function of the

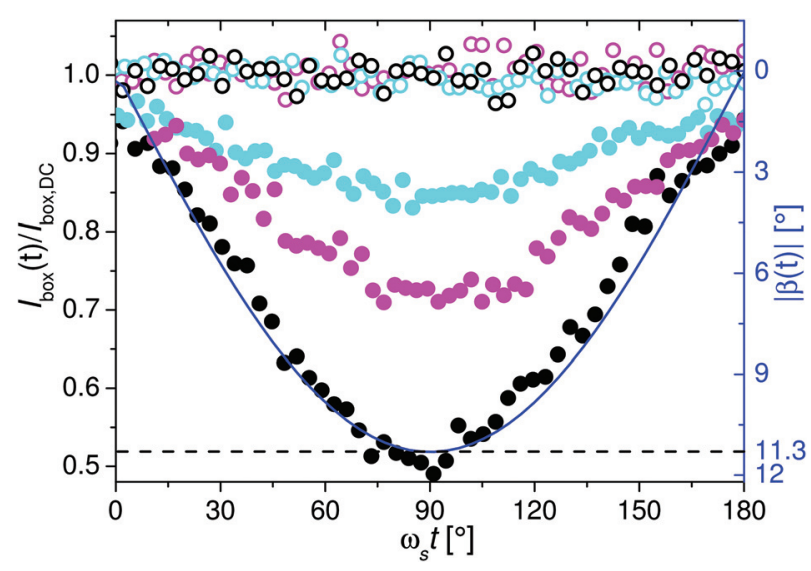

Fig. 9 Integrated intensities for sample frequencies of $\omega_{\mathrm{s}}=157.1 \mathrm{~Hz}$ (black), $\omega_{\mathrm{s}}=11309.7 \mathrm{~Hz}$ (magenta) and $\omega_{\mathrm{s}}=18849.6 \mathrm{~Hz}$ (cyan) in the horizontal sector (full symbols) as well as in the vertical sector (open symbols) as a function of time. The integrated intensities were normalized to the sector area and divided by the normalized integrated intensity $I_{\text {box,DC }}$ of the static scattering pattern in the circular area indicated in Fig. 6. Phase shifts between the signal and the magnetic field were omitted by shifting the intensity on the time-scale, so that $\omega_{s} t=90^{\circ}$ corresponds to the minimum and hence maximal rotation of the nanorod ensemble out of the neutron beam. The blue line is the orientation of the magnetic field vector with respect to the neutron beam as a function of time. 
measured integrated intensity for $\omega_{\mathrm{s}}=157.1 \mathrm{~Hz}$, was fitted with a phenomenological polynomial (Fig. 10). This calibration enabled to translate measured integrated intensities to $|\zeta(t)|$ (Fig. 11) and to determine the oscillation amplitude as function of $\omega_{\mathrm{s}}$. The results of the given examples are for $\omega_{\mathrm{s}}=$ $11309.7 \mathrm{~Hz} \zeta_{0}=7.8^{\circ}$ and for $\omega_{\mathrm{s}}=18849.6 \mathrm{~Hz} \zeta_{0}=5.5^{\circ}$ (Fig. 11). A summary of the complete set for all measured frequencies is shown in Fig. 4. Comparison with the results of the OT shows a shift to smaller frequencies by a factor of 1.5 . For this observation two possible explanations can be envisaged. First, the volume concentration $V_{\text {mag }} / V$ of the colloid characterized by SANS was significantly larger compared to OT

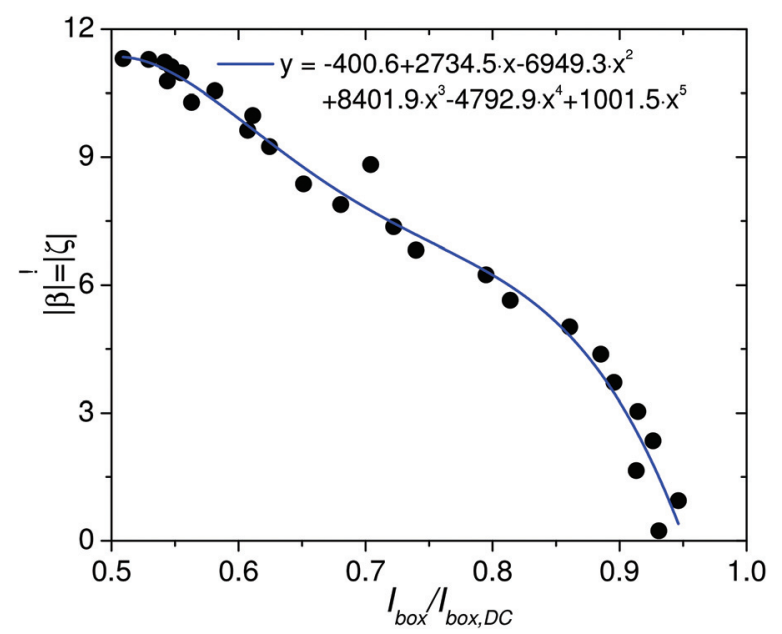

Fig. 10 Magnitude $|\beta|$ of the orientation angle of the field vector and hence the symmetry axis of the ensemble $|\zeta|$ as a function of the integrated intensity at $\omega_{\mathrm{s}}=157.1 \mathrm{~Hz}$ (black). The fitting function enables to translate measured intensities into $|\zeta|$.

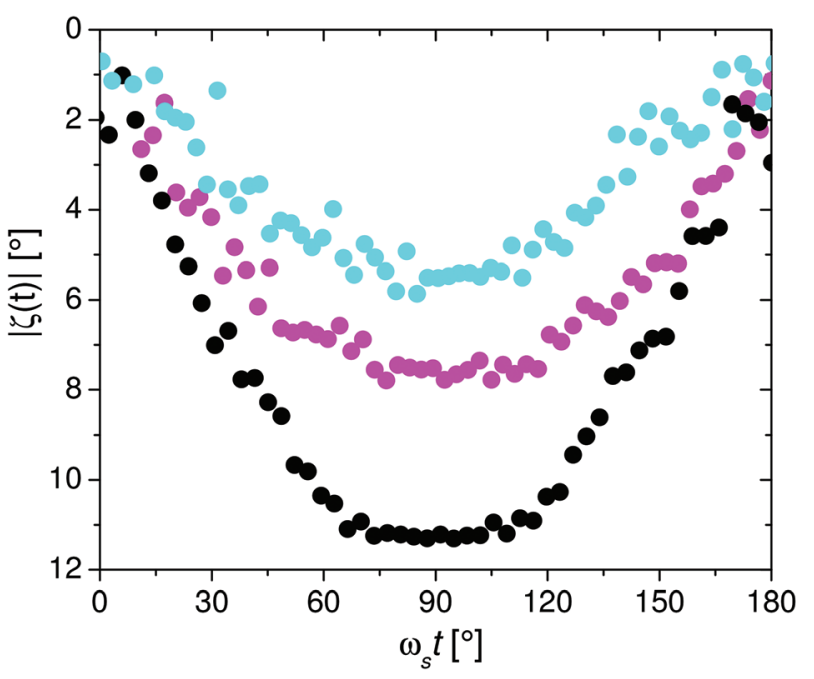

Fig. 11 Orientation of the symmetry axis of the nanorod ensemble with respect to the neutron beam for $\omega_{\mathrm{s}}=157.1 \mathrm{~Hz}$ (black), $\omega_{\mathrm{s}}=11309.7 \mathrm{~Hz}$ (magenta) and $\omega_{\mathrm{s}}=18849.6 \mathrm{~Hz}$ (cyan) as a function of time.
$\left(V_{\mathrm{mag}}^{\mathrm{SANS}} / V=100 \times V_{\mathrm{mag}}^{\mathrm{OT}} / V=0.5 \%\right.$, which could result in hydrodynamic interactions between the oscillating nanorods. Second, using the integrated intensity from the measurement with $\omega_{\mathrm{s}}=157.1 \mathrm{~Hz}$ as reference, it was implicitly assumed that the orientation distribution $P(\Theta)$ does not change with increasing frequency, identical to the data analysis of the OT measurements. However, if $P(\Theta)$ changes occur with increasing frequency, the data analysis would be incorrect and thus the obtained values for the oscillation amplitude by OT and SANS (Fig. 4) should not agree. Therefore, in the following, we focus on the time-dependent intensities $I(Q)$ of the sectors in order to get insight into the dynamic orientation distribution of the nanorod ensemble.

As already shown in Fig. 9 the integrated intensity in the vertical sector for each frequency is constant and identical to the static case. Furthermore, the functional form of $I(Q)$ is also identical to the circular averaged intensity of the ensemble aligned in the static field, as exemplarily shown for $\omega_{\mathrm{s}}=157.1$ $\mathrm{Hz}$ in Fig. 7. Therefore, it can be already concluded, that orientation distribution along the $y$-direction does not change with increasing frequency.

In Fig. $7 I(Q)$ of the horizontal sector of the time channel with minimal integrated intensity for the frequency $\omega_{\mathrm{s}}=157.1$ $\mathrm{Hz}$ is displayed (black full circles). In this time channel the nanorod ensemble should be quasistatically (low frequency) rotated by the maximum angle $\zeta=11.3^{\circ}$ out of the neutron beam ( $z^{\prime}$-direction, Fig. 8). Under assumption of a coherent rotation of the entire ensemble (represented by the characteristic angle $[\Theta]=12^{\circ}$ and an symmetric distribution in $\Phi$ around the symmetry axis) the expected scattering intensity $I(Q)$ (eqn (4)) in thehorizontal sector ( $x^{\prime}$-direction) is proportional to the form factor $F\left(Q, \mu_{x^{\prime}}\right)$ (eqn (5) and (10)). Simulation of the orientation averaged SANS intensity (eqn (7)) resulted in good agreement with the experimental data, as shown in Fig. 7. This indicates that at a frequency of $\omega_{\mathrm{s}}=157.1$ $\mathrm{Hz}$ the orientation distribution of the rods around the symmetry axis at the maximum rotation angle is comparable to the static case.

In Fig. 12 and 13, the radially averaged intensity of the time channels with minimal integrated intensity $\left(\omega_{\mathrm{s}} t=90^{\circ}\right)$ for $\omega_{\mathrm{s}}=$ $11309.7 \mathrm{~Hz}$ and $\omega_{\mathrm{s}}=18849.6 \mathrm{~Hz}$ are plotted, respectively. Comparison with $I(Q)$ of the time channels from the measurement at $\omega_{\mathrm{s}}=157.1 \mathrm{~Hz}$ with equal integrated intensities (i.e. equal average orientation) shows, that they are virtually identical. Additionally, the simulated intensities $I(Q)$ for rotation angles $\zeta=7.8^{\circ}$ and $5.5^{\circ}$ were in good agreement with the experimental data for $\omega_{\mathrm{s}}=11309.7 \mathrm{~Hz}$ and $18849.6 \mathrm{~Hz}$, respectively. In other words, the increasing value of the minimum integrated intensity is essentially the result of a reduced average oscillation amplitude of the nanorod ensemble at increasing frequency without a significant change in the orientation distribution function.

Overall it can be concluded that the orientation distribution of the rods around the symmetry axis is independent on the oscillation frequency up to $20 \mathrm{kHz}$ and comparable to the static case. As a consequence, the mean-field approach intro- 


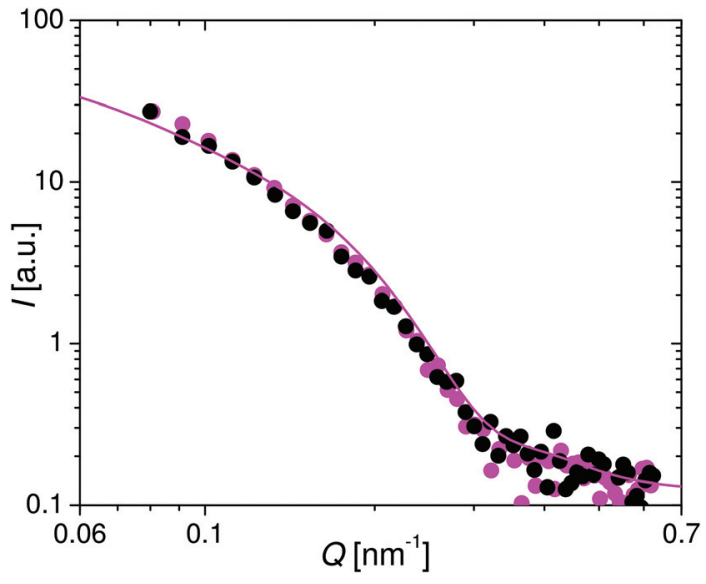

Fig. 12 Intensity after radially averaging in the horizontal sector of the time channel with minimal integrated intensity at $\omega_{\mathrm{s}}=11309.7 \mathrm{~Hz}$ (magenta) and of the time channel with identical integrated intensity at $\omega_{\mathrm{s}}=157.1 \mathrm{~Hz}$ (black, see Fig. 9). Full line is the simulated intensity of the ensemble when the symmetry axis is rotated by $7.8^{\circ}$ out of the neutron beam.

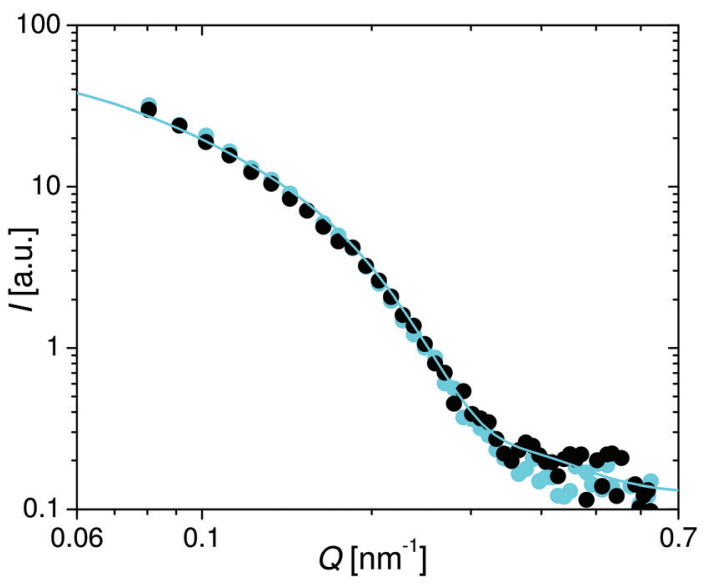

Fig. 13 Intensity after radially averaging in the horizontal sector of the time channel with minimal integrated intensity at $\omega_{\mathrm{s}}=18849.6 \mathrm{~Hz}$ (cyan) and of the time channel with identical integrated intensity at $\omega_{\mathrm{s}}=$ $157.1 \mathrm{~Hz}$ (black, see Fig. 9). Full line is the simulated intensity of the ensemble when the symmetry axis is rotated by $5.5^{\circ}$ out of the neutron beam.

duced in ref. 6 to analyze the oscillation behavior of such nanorod ensembles in oscillating magnetic fields is justified. This also means, that the observed shift (Fig. 4) of the average oscillation amplitude of the nanorod ensemble to lower frequencies detected by SANS in comparison to OT, must be real. As previously mentioned, a possible explanation for this observation may be the significantly higher volume concentration $V_{\text {mag }} / V$ of the colloid characterized by SANS compared to OT $\left(V_{\mathrm{mag}}^{\mathrm{SANS}} / V=100 \times V_{\mathrm{mag}}^{\mathrm{OT}} / V=0.5 \%\right.$ ), which could lead to hydrodynamic interactions between the nanorods. To define an upper limit for the volume concentration, or rather for the average interparticle distance, so that such hydrodynamic interactions are negligible, would require a systematic investigation. However, it has to be emphasized that a possible structure formation (e.g. chains) in the concentrated nanorod colloid induced by dipolar interactions can be ruled out here, as the scattering intensity could be simulated in all cases by a dilute dispersion (no structure factor) of cylinders with the length and diameter distributions determined by TEM.

\section{Conclusions}

The response of a colloidal dispersion of $\mathrm{Ni}$ nanorods to an oscillating magnetic field was characterized by SANS at the instrument D22 at the ILL, Grenoble, using the TISANE mode in the frequency range of $0 \mathrm{~Hz} \leq \omega_{\mathrm{s}} \leq 18849.6 \mathrm{~Hz}$. In the static case the scattering intensity can be well described by the cylinder form factor using the structural parameters determined by TEM and VSM. An oscillation of the field vector resulted in a time-dependency of the SANS intensity due to the shape anisotropy of the magnetic nanorods. Analysis of the nuclear scattering data revealed that in the range of low frequencies (up to $\omega_{\mathrm{s}}=157.1 \mathrm{~Hz}$ ) the oscillation of the nanorod ensemble is in phase with the field vector and that the orientation distribution around the symmetry axis is comparable to the static case. With increasing frequency, the oscillation of the horizontal SANS intensity decreased due to damping of the nanorod rotation as a result of an increased viscous drag. The central result of the analysis of the scattering data in the high-frequency range is that despite the viscous drag and the polydispersity of the ensemble no change of the orientation distribution was observed. Consequently, the relaxation behavior detected by means of ensemble averaging methods (e.g. optical transmission measurements as additionally done in this study) can be correctly analyzed by mean field approaches, confirming the oscillating field method as a powerful alternative for nanosensing approaches.

\section{Acknowledgements}

We acknowledge the ILL for provision of neutron beamtime at the D22 and the Bundesministerium für Bildung und Forschung (BMBF) for financial support (Project: 05K10TS1). A. Michels acknowledges financial support from the FNR (Project No. INTER/DFG/12/07).

\section{References}

1 C. Barrera, V. Florian-Algarin, A. Acevedo and C. Rinaldi, Soft Matter, 2010, 6, 3662-3668.

2 V. L. Calero-DdelC, D. I. Santiago-Quinonez and C. Rinaldi, Soft Matter, 2011, 7, 4497-4503.

3 N. Frickel, R. Messing and A. M. Schmidt, J. Mater. Chem., 2011, 21, 8466-8474.

4 E. Roeben, L. Roeder, S. Teusch, M. Effertz, U. Deiters and A. Schmidt, Colloid Polym. Sci., 2014, 292, 2013-2023. 
5 A. Günther, P. Bender, A. Tschöpe and R. Birringer, J. Phys.: Condens. Matter, 2011, 23, 325103.

6 A. Tschöpe, K. Birster, B. Trapp, P. Bender and R. Birringer, J. Appl. Phys., 2014, 116, 184305.

7 J. Connolly and T. G. S. Pierre, J. Magn. Magn. Mater., 2001, 225, 156-160.

8 A. P. Astalan, F. Ahrentorp, C. Johansson, K. Larsson and A. Krozer, Biosens. Bioelectron., 2004, 19, 945-951.

9 S. H. Chung, A. Hoffmann, S. D. Bader, C. Liu, B. Kay, L. Makowski and L. Chen, Appl. Phys. Lett., 2004, 85, 29712973.

10 C.-Y. Hong, C. C. Wu, Y. C. Chiu, S. Y. Yang, H. E. Horng and H. C. Yang, Appl. Phys. Lett., 2006, 88, 212512.

11 M. Strömberg, J. Göransson, K. Gunnarsson, M. Nilsson, P. Svedlindh and M. Stromme, Nano Lett., 2008, 8, 816-821.

12 F. Öisjöen, J. F. Schneiderman, A. P. Astalan, A. Kalabukhov, C. Johansson and D. Winkler, Biosens. Bioelectron., 2010, 25, 1008-1013.

13 K. Enpuku, Y. Tamai, T. Mitake, T. Yoshida and M. Matsuo, J. Appl. Phys., 2010, 108, 034701.

14 J. Dieckhoff, T. Yoshida, K. Enpuku, M. Schilling and F. Ludwig, IEEE Trans. Magn., 2012, 48, 3792-3795.

15 T. Z. Gómez de la Torre, R. Ke, A. Mezger, P. Svedlindh, M. Stromme and M. Nilsson, Small, 2012, 8, 2174-2177.

16 A. C. Bohorquez and C. Rinaldi, Part. Part. Syst. Charact., 2014, 31, 561-570.

17 R. S. Bejhed, T. Z. G. de la Torre, M. Donolato, M. F. Hansen, P. Svedlindh and M. Strömberg, Biosens. Bioelectron., 2015, 66, 405-411.

18 A. Ranzoni, J. J. H. B. Schleipen, L. J. van IJzendoorn and M. W. J. Prins, Nano Lett., 2011, 11, 2017-2022.

19 S. Schrittwieser, F. Ludwig, J. Dieckhoff, K. Soulantica, G. Viau, L.-M. Lacroix, S. M. Lentijo, R. Boubekri, J. Maynadié, A. Hütten, H. Brückl and J. Schotter, ACS Nano, 2012, 6, 791-801.

20 S. Schrittwieser, F. Ludwig, J. Dieckhoff, A. Tschöpe, A. Günther, M. Richter, A. Hütten, H. Brückl and J. Schotter, Small, 2014, 10, 407.

21 J. Dieckhoff, A. Lak, M. Schilling and F. Ludwig, J. Appl. Phys., 2014, 115, 024701.

22 C. A. Ross, M. Hwang, M. Shima, J. Y. Cheng, M. Farhoud, T. A. Savas, H. I. Smith, W. Schwarzacher, F. M. Ross, M. Redjdal and F. B. Humphrey, Phys. Rev. B: Condens. Matter, 2002, 65, 144417.
23 A. Günther, S. Monz, A. Tschöpe, R. Birringer and A. Michels, J. Magn. Magn. Mater., 2008, 320, 1340-1344.

24 P. Bender, A. Günther, A. Tschöpe and R. Birringer, J. Magn. Magn. Mater., 2011, 323, 2055-2063.

25 P. Bender, F. Krämer, A. Tschöpe and R. Birringer, J. Phys. D: Appl. Phys., 2015, 48, 145003.

26 T. Yoshida, K. Enpuku, J. Dieckhoff, M. Schilling and F. Ludwig, J. Appl. Phys., 2012, 111, 053901.

27 A. Satoh, J. Colloid Interface Sci., 2001, 234, 425-433.

28 M. Aoshima, A. Satoh, R. W. Chantrell and G. N. Coverdale, J. Colloid Interface Sci., 2002, 253, 455-464.

29 A. Satoh, M. Ozaki, T. Ishikawa and T. Majima, J. Colloid Interface Sci., 2005, 292, 581-590.

30 D. Soto-Aquino and C. Rinaldi, Phys. Rev. E: Stat. Phys., Plasmas, Fluids, Relat. Interdiscip. Top., 2010, 82, 046310.

31 K. Nielsch, F. Müller, A.-P. Li and U. Gösele, Adv. Mater., 2000, 12, 582-586.

32 O. P. Watts, Trans. Am. Electrochem. Soc., 1916, 29, 395.

33 H. Masuda and M. Satoh, Jpn. J. Appl. Phys., 1996, 35, L126-L129.

34 R. Skomski, J. Phys.: Condens. Matter, 2003, 15, R841.

35 T. Klein, A. Laptev, A. Günther, P. Bender, A. Tschöpe and R. Birringer, J. Appl. Phys., 2009, 106, 114301.

36 P. Song, D. Wen, Z. X. Guo and T. Korakianitis, Phys. Chem. Chem. Phys., 2008, 10, 5057-5065.

37 R. Karmhag, G. A. Niklasson and M. Nygren, J. Appl. Phys., 2001, 89, 3012-3017.

38 C. P. Bean and J. D. Livingston, J. Appl. Phys., 1959, 30, S120-S129.

39 R. Gähler and R. Golub, ILL SC, 1999, 99, 73.

40 A. Wiedenmann, U. Keiderling, K. Habicht, M. Russina and R. Gähler, Phys. Rev. Lett., 2006, 97, 057202.

41 http:/www.ill.eu/instruments-support/instrument-groups/ groups/lss/grasp/home/.

42 P. Debye, Polar Molecules, New York, Dover, 1945.

43 J. S. Pedersen, Adv. Colloid Interface Sci., 1997, 70, 171210.

44 Modern Techniques for Characterizing Magnetic Materials, ed. Y. Zhu, Springer, US, 2005, pp. 107-155.

45 G. Fournet, Bull. Soc. Fr. Mineral. Cristallogr.., 1951, 74, 39113.

46 T. Maurer, F. Zighem, S. Gautrot, F. Ott, G. Chaboussant, L. Cagnon and O. Fruchart, Phys. Procedia, 2013, 42, 74-79. 